\title{
Effect of microstructure on dynamic compressive behavior of cellular materials
}

\author{
Kohei Tateyama ${ }^{1 *}$, and Keiko Watanabe ${ }^{1}$ \\ ${ }^{1}$ Department of Mechanical Engineering, Ritsumeikan University, Shiga 525-8577, Japan
}

\begin{abstract}
It is known that the microstructure of cellular materials has a significant impact on their compressive properties. To study these phenomena, a hierarchical Poisson disk sampling algorithm and Voronoi partitioning were used to create a 3D numerical analysis model of cellular materials. In this study, we prepared random, periodic, and ellipsoidal cell models to investigate the effects of cell shape randomness and oblateness. Numerical experiments were performed using the finite element method solver RADIOSS. In the numerical analysis, an object collided with the cellular materials at a velocity of $25 \mathrm{~m} / \mathrm{s}$. The results showed that the flow stress of the random cell model was higher than that of the periodic cell model. Further, it was found that the aspect ratio of the cell shape has a significant impact on the mechanical properties of cellular materials.
\end{abstract}

\section{Introduction}

Since cellular materials are lightweight and have high energy absorption ability, they are widely used in applications such as packing materials to reduce dynamic and impact loads. A major feature of cellular materials is that the structure contains many pores, and the shape of the cells affects the mechanical properties of the structure. Although many studies have been conducted on the mechanical properties of cellular materials [1, 2], few studies investigate the effect of microstructure on mechanical properties both experimentally and analytically. A thorough examination of the effect of microstructure is required to understand the mechanical properties of cellular materials accurately. Recently, a methodology was proposed to generate the 3D geometry of closed-cell metallic foams with detailed control of their microstructural and morphological features [3-6]. However, this methodology does not consider the anisotropy of the cell shape. Therefore, in this study, we created a numerical analysis model of the cellular materials in which the cell structure was precisely controlled using the finite element method (FEM) and analytically evaluated the effect of the microstructure on the mechanical properties of the cellular materials.

\section{Numerical experiment}

\footnotetext{
* Corresponding author: koheit@fc.ritsumei.ac.jp
} 


\subsection{Numerical analysis model}

\subsubsection{Random cell model}

In this study, the hierarchical Poisson disk sampling method [7] was used as the cell matrix (mother points) generation method. The space is divided using Voronoi partitioning for the generated mother points. Subsequently, a numerical analysis model (cell model) was created using the dividing surface as the cell wall. The shape of the cell model was a cube with a side of $100 \mathrm{~mm}$, the number of cells was 600 , and the relative density was $100 \mathrm{~kg} / \mathrm{m}^{3}$. Figure 1 (a) shows the basic random cell model created using this procedure. Based on this random cell model, we created a periodic cell model with periodicity and an ellipsoidal cell model with oblateness.

\subsubsection{Periodic cell model}

A periodic cell model was prepared to evaluate the effect of the randomness of the cell shape, as shown in Fig. 1(b). Periodic and random cell models have the same parameters, except for periodicity. The periodic cell model has five times greater periodicity on all the axes. This model is similar to the rule model as the periodicity increases, but it should be noted that it is different from the rule model.

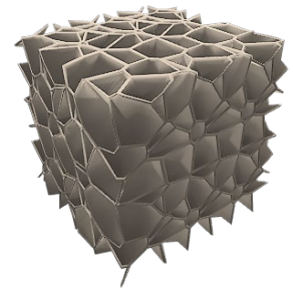

(a)

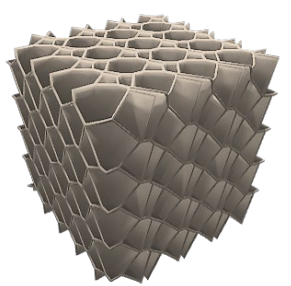

(b)

Fig.1. External view of (a) random cell model, and (b) periodic cell model.

\subsubsection{Ellipsoidal cell model}

Three types of models were prepared to evaluate the effect of the aspect ratio of the cell shape: random cell model (aspect ratio 1.0), vertical ellipsoidal cell model (VE model: aspect ratio 0.5 ), and horizontal ellipsoidal cell model (HE model: aspect ratio 1.5), as shown in Fig. 2. These models have the same density. In particular, VE and HE cell models have the same cell shape and are different only in terms of the direction.

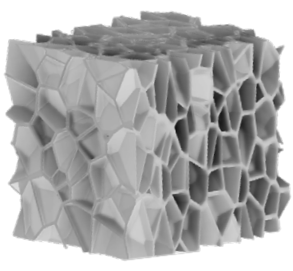

(a)

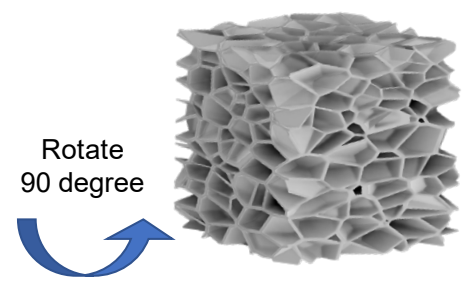

(b)

Fig.2. External view of (a) vertical ellipsoidal cell model, and (b) horizontal ellipsoidal cell model. 


\subsection{Numerical analysis condition}

A numerical experiment was performed using the FEM solver RADIOSS (Altair Engineering). The model consists of an upper rigid wall, a lower rigid wall, and a cell wall. Figure 3 shows the configuration of the analysis model using the random cell model. The cell wall was meshed using solid elements. The material properties of the cell wall were set as typical properties of pure aluminum. The Johnson-Cook model was used for the constitutive equation, and the parameters are listed in Table 1. Compressive numerical experiments were performed by applying a constant velocity to the upper rigid wall. The constant velocity was $25 \mathrm{~m} / \mathrm{s}$ (initial strain rate: $2.5 \times 10^{2} \mathrm{~s}^{-1}$ ). A compressive load was obtained for the upper and lower rigid walls.

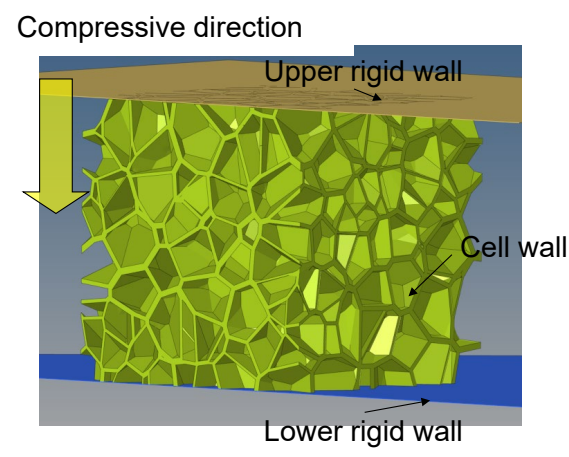

Fig.3. Configuration of numerical analysis model using the random cell model.

Table 1. Material property parameters of pure aluminum

\begin{tabular}{|c|c|c|}
\hline Reference density & 2700 & $\mathrm{~kg} / \mathrm{m}^{3}$ \\
Equation of state & Linear & - \\
Bulk modulus & 57.25 & $\mathrm{GPa}$ \\
Material Strength Model & Johnson Cook & - \\
Initial yeild Stress (A) & 115.8 & $\mathrm{MPa}$ \\
Hardening Constant (B) & 68.5 & $\mathrm{MPa}$ \\
Hardening Exponent (n) & 0.58 & - \\
Strain Rate Constant (C) & 0.016 & - \\
Thermal Softening Exponent & 1.13 & $\mathrm{~K}$ \\
Melting Temperature & 933 & \\
\hline
\end{tabular}

\section{Result and discussion}

Figure 4 shows the stress-strain relationships of the random and periodic cell models obtained from the numerical experiment. In general, cellular materials exhibit three phases of compressive deformation: linear-elastic response, plateau deformation, and densification [7]. Using the model developed in this study, three phases of compressive deformation were observed. The flow stress tended to be different because of the difference in the start timing of the plastic deformation. 


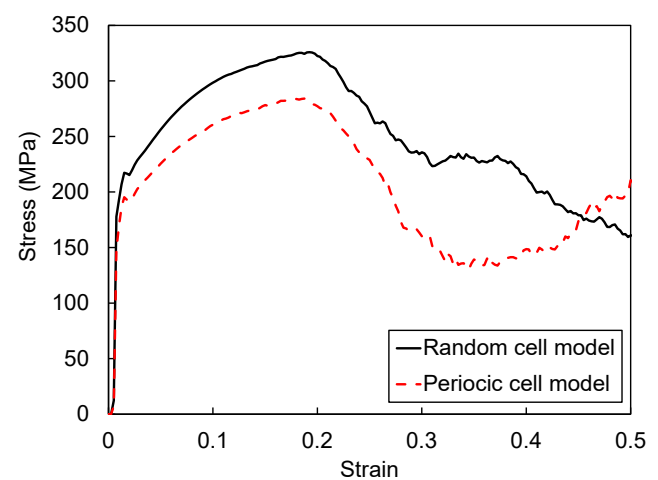

Overall view

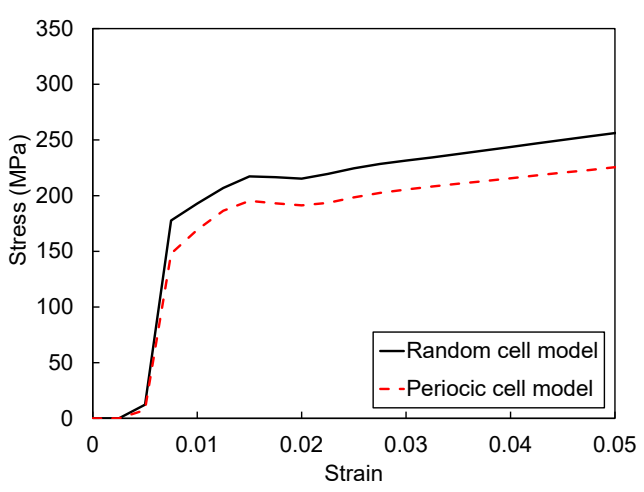

Enlarged view

Fig.4. Stress--strain relationships of random and periodic cell models.
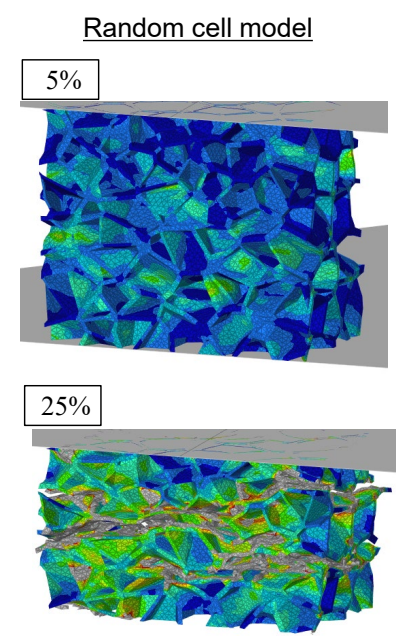

Periodic cell model
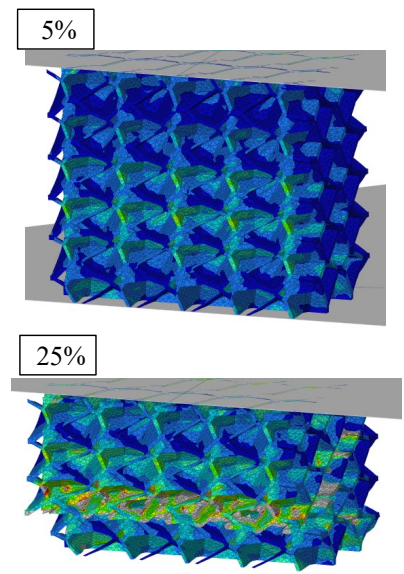

Contour : Equivalent plastic strain of cell wall

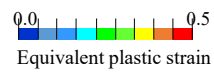

Fig.5. Deformation behavior of random and periodic cell models at $5 \%$ and $25 \%$ compression.

To investigate this tendency, Fig. 5 shows the deformation behavior of each model. In the random cell model, the deformation area was wide. It is presumed that this is because the deformed area has expanded owing to the dispersion of the weak and strong parts. Macroscopically, the strong part reinforces the weak part. In contrast, in the periodic cell model, it can be confirmed that the deformation is local. This is because the strong and weak parts are repeated regularly, and the weak parts are emphasized. This tendency does not occur with a perfectly regular model. From the above, it can be seen that the perfectly regular cell model and periodic cell model are different. It seems that the slight differences behave like early flaws in the periodic cell model.

Figure 6 shows the stress-strain relationships of the random, VE, and HE cell models obtained from the numerical experiment. Focusing on the elastic response regions of the three models, although the three models have the same conditions except for the cell aspect ratio, there is a clear difference in the slope of the elastic response. In the VE cell model, an elastic response with a large slope was clearly observed at the initial stage of deformation compared 


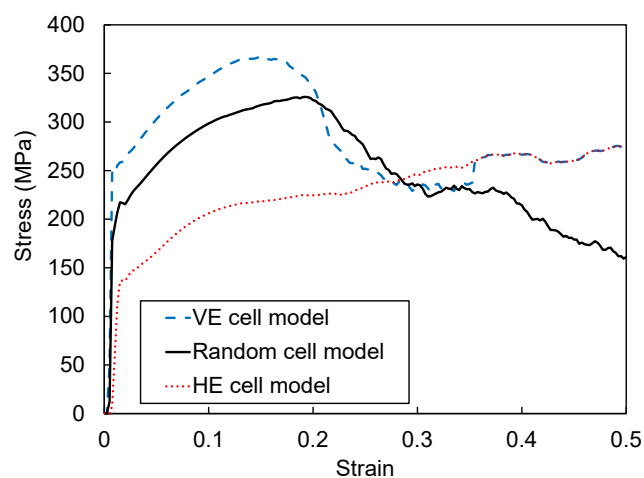

Overall view

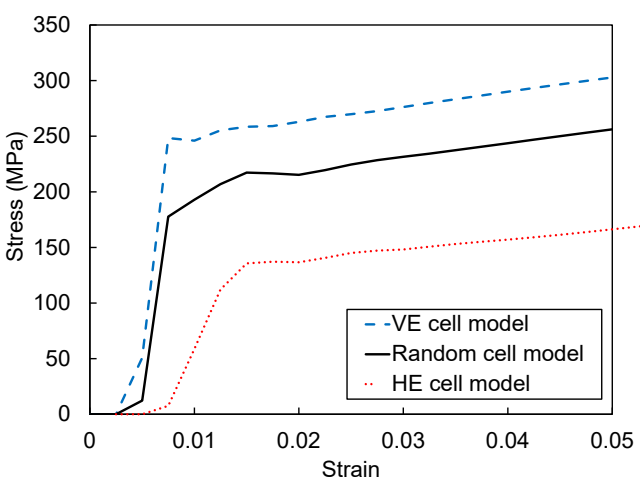

Enlarged view

Fig.6. Stress-strain relationships of random, VE, and HE cell models.
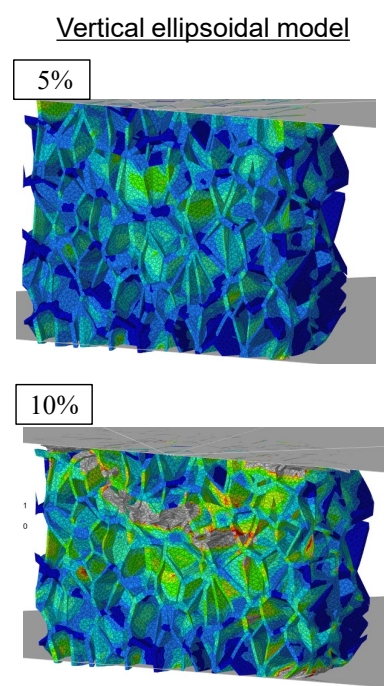

Horizontal ellipsoidal model
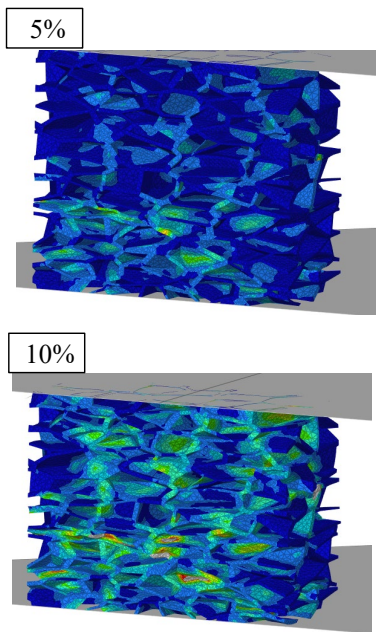

Contour : Equivalent plastic strain of cell wall

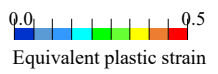

Fig.7. Deformation behavior of VE and HE cell models at 5\% and 10\% compression.

with the other models. In contrast, the gradient of the elastic response tended to decrease compared to that of the random and HE cell models. To investigate this tendency, the deformation behavior of each model was observed (Fig. 7). In the VE cell model, because the curvature of the cell wall was large, bending deformation was dominant. In contrast, in the random and HE cell models, it was estimated that the compressive deformation mainly occurred during the initial stage of deformation.

Thus, it was confirmed that when the aspect ratio of the cell shape was different, the deformation mode changed owing to the difference in the curvature of the cell wall at the initial stage of the deformation. It was found that the difference in the deformation mode affected the slope of the elastic response. From this result, it was clarified that the cell shape oblateness significantly affects the compressive properties of cellular materials. 


\section{References}

1. L.J. Gibson, M.F. Ashby, Cellular Solids (Cambridge University Press, Cambridge, 1997)

2. Y. Sun, Q.M. Li, Int. J. Impact Eng. 112, 74 (2018)

3. A. Ghazi, P. Berke, K. Ehab Moustafa Kamel, B. Sonon, C. Tiago, T.J. Massart, Int. J. Eng. Sci. 143, 92 (2019)

4. A. Ghazi, C. Tiago, B. Sonon, P. Berke, T.J. Massart, Int. J. Mech. Sci. 168, 105298 (2020)

5. N.G. Kilingar, K. Ehab Moustafa Kamel, B. Sonon, T.J. Massart, L. Noels, Eur. J. Mech. A-Solid 78, 103847 (2019)

6. K. Ehab Moustafa Kamel, B. Sonon, T.J. Massart, Compt. Mech. 64, 1049 (2019)

7. M. D. Mccool, Hierarchical Poisson disk sampling distributions, in Proceedings of Graphics Interface, May 1992, Vancouver, British Columbia, Canada (1992) 